\title{
The primary pretenders
}

by

John H. Conway (Princeton, N.J.), Richard K. Guy (Calgary, Alta.), W. A. Schneeberger (Princeton, N.J.) and N. J. A. SloAne (Murray Hill, N.J.)

Perhaps the most famous theorem in number theory is Fermat's theorem. Not Fermat's Last Theorem, of course, because that is now old hat, but Fermat's Little Theorem:

If $p$ is a prime, and $b$ is a positive integer prime to $p$, then

$$
b^{p-1} \equiv 1(\bmod p),
$$

which we prefer to write in the simpler form

$$
b^{p} \equiv b(\bmod p) .
$$

If the converse of the theorem were true, then number theory would be a lot simpler than it is, but fortunately that is not the case. Counterexamples to the converse of the first (and, very occasionally, the second) form of Fermat's theorem are called pseudoprimes. A well-known example is $341=$ $11 \cdot 31$, which is a pseudoprime to base 2 :

$$
2^{340} \equiv 1(\bmod 341) .
$$

The literature on pseudoprimes is extensive; for an introduction see Section A12 of the second author's Unsolved Problems in Number Theory, 2nd ed., Springer, 1994. D. H. Lehmer found the even pseudoprime $161038=$ $2 \cdot 73 \cdot 1103$ and N. G. W. H. Beeger showed that there were infinitely many.

The Carmichael numbers, such as $561=3 \cdot 11 \cdot 17$, are counterexamples to the second form of Fermat's theorem to any base:

$$
b^{561} \equiv b(\bmod 561), \quad b=1,2, \ldots
$$

The second form of the theorem admits a much wider class of counterexamples than the first, and to distinguish them from the pseudoprimes we will call any composite number $q$ such that $b^{q} \equiv b(\bmod q)$ a prime pretender to base $b$. 
We investigate $q_{b}$, the least prime pretender, or primary pretender, for the base $b$.

We will see that there are only 132 distinct primary pretenders, and that $q_{b}$ is a periodic function of $b$ whose period is the 122-digit number

19568584333460072587245340037736278982017213829337604336734362 294738647777395483196097971852999259921329236506842360439300

What is this number? Well, it is $p !_{59} p !_{9}$, where $p !_{k}$ is the product of the first $k$ primes, $p_{1} p_{2} \ldots p_{k}$. And where do $p_{59}$ and $p_{9}$ come from? $p_{59}=277$ is the largest possible prime factor, and $p_{9}=23$ is the largest possible repeated prime factor, of a composite number less than the Carmichael number 561.

For what bases is 4 a prime pretender? If $b \equiv 0,1,2,3(\bmod 4)$, then $b^{4} \equiv 0,1,0,1$, so 4 is a prime pretender just for $b \equiv 0,1(\bmod 4)$.

The similar calculations mod 6 and 8 show that 6 is a prime pretender for bases $\equiv 0$ or $1(\bmod 3)$ and that 8 is a prime pretender for bases $\equiv 0$ or 1 $(\bmod 8)$. It follows that every number for which 8 is a prime pretender also has 4 as a prime pretender, so that 8 can never be the primary pretender. The calculations mod 9 show that 9 is a prime pretender for bases $\equiv 0$, 1 or $8(\bmod 9)$, which may also be described as the square roots of 0 or $1(\bmod 9)$.

These results can be recorded by saying that for $q=4$ and 9 ,

" $q$ is a prime pretender just for the bases

that are $k$ th roots of 0 or $1 \bmod m "$

for a certain $k$ and $m$. (It will turn out that such an assertion holds for all the primary pretenders - see Table 3.) They imply that we know the primary pretender $q_{b}$ for all but the four residue classes 2, 11, 14, $23(\bmod$ $36)$ :

\begin{tabular}{|c|c|c|c|c|c|c|c|c|c|c|c|c|c|c|c|c|c|c|}
\hline$b$ & $\equiv 0$ & 1 & 2 & 3 & 4 & 5 & 6 & 7 & 8 & 9 & 10 & 11 & 12 & 13 & 14 & 15 & & 17 \\
\hline$q_{b}$ & $=4$ & 4 & $?$ & 6 & 4 & 4 & 6 & 6 & 4 & 4 & 6 & $?$ & 4 & 4 & $?$ & 6 & 4 & 4 \\
\hline$b$ & $\equiv 18$ & 19 & 20 & 21 & 22 & 23 & 24 & 25 & 26 & 27 & 28 & 29 & 30 & 31 & 32 & 33 & & 35 \\
\hline$q_{b}$ & $=$ & 6 & 4 & 4 & 6 & ? & 4 & 4 & 9 & 6 & 4 & 4 & 6 & 6 & 4 & 4 & 6 & 9 \\
\hline
\end{tabular}

The values of $q_{b}$ up to 21 for the residue classes mod 1260 missing from the last display are given in Table 1 . In fact, $q_{b} \geq 22$ for just the 32 residue classes mod 1260 indicated by ? in Table 1.

The number of distinct values of $q_{b}$ is bounded, since the Carmichael number 561 will always serve if no smaller exponent has been found. The other numbers which occur are products of just two prime factors: twice the primes from 2 to 277 ; thrice the primes from 3 to 181; five times those primes which are $\equiv 1(\bmod 4)$ from 5 to 109 ; seven times those primes which 
Table 1. $q_{b}=10,14,15,21$ for just 108 residue classes mod 1260

\begin{tabular}{r|cccccccccccccccccccccc}
\hline$b=$ & 2 & 11 & 14 & 23 & 38 & 47 & 50 & 59 & 74 & 83 & 86 & 95 & 110 & 119 & 122 & 131 & 146 & 155 & 158 & 167 \\
\hline+0 & $?$ & 10 & 14 & $?$ & $?$ & $?$ & 10 & 15 & 15 & 21 & 10 & 10 & 10 & 14 & $?$ & 10 & 10 & 10 & $?$ & 21 \\
+180 & 14 & 10 & 15 & 14 & 14 & $?$ & 10 & 14 & 15 & $?$ & 10 & 10 & 10 & 15 & 14 & 10 & 10 & 10 & $?$ & $?$ \\
+360 & $?$ & 10 & 15 & $?$ & 21 & 14 & 10 & 15 & 14 & $?$ & 10 & 10 & 10 & 15 & 21 & 10 & 10 & 10 & 14 & $?$ \\
+540 & $?$ & 10 & 14 & $?$ & $?$ & 21 & 10 & 15 & 15 & 14 & 10 & 10 & 10 & 14 & $?$ & 10 & 10 & 10 & $?$ & 14 \\
+720 & 14 & 10 & 15 & 14 & $?$ & $?$ & 10 & 15 & 15 & $?$ & 10 & 10 & 10 & 15 & $?$ & 10 & 10 & 10 & $?$ & $?$ \\
+900 & 21 & 10 & 15 & 21 & 14 & $?$ & 10 & 14 & 14 & $?$ & 10 & 10 & 10 & 15 & 14 & 10 & 10 & 10 & 14 & $?$ \\
+1080 & $?$ & 10 & 15 & $?$ & $?$ & 14 & 10 & 15 & 15 & 14 & 10 & 10 & 10 & 15 & $?$ & 10 & 10 & 10 & 21 & 14 \\
\hline
\end{tabular}

are $\equiv 1(\bmod 3)$ from 7 to 79 ; eleven times 11,31 and 41; thirteen times 13 and 37 ; and the squares of 17,19 and 23.

Computer calculations of the numbers in the missing residue classes for values of $b$ up to 50000 appear in Table 2; the numbers at the left show the multiples of 1260 to be added. The programs used to calculate Tables 2 and 3 were straightforward, essentially using brute force.

Our final table, Table 3, shows how long it takes before any particular value of $q_{b}$ appears; it can be summarized as follows. The value of $q_{b}$ is

$$
\begin{aligned}
& 4 \quad \text { if } b \equiv 0,1(\bmod 4) \\
& \text { else } 6 \text { if } b \equiv 0,1(\bmod 3) \\
& \text { else } \quad 9 \quad \text { if } b \equiv 8 \quad(\bmod 9) \\
& \text { else } \quad \ldots \quad \ldots \quad \ldots \quad \ldots \\
& \text { else } 561 \quad \text { if } b \equiv 0 \quad(\bmod 1)
\end{aligned}
$$

where the various statements can all be put into the form

$$
\text { "else } q \text { if } b \text { is a } k \text { th root of } 0 \text { or } 1(\bmod m) \text { " }
$$

for appropriate values of $q, k$ and $m$. The table also gives the first base, that is, the least $b$ for which $q_{b}=q$, and the rarity $r$ of $q$, meaning that $q$ is the primary pretender for 1 in every $r$ bases. For example

$$
25 \quad 4 \operatorname{th}(25) \quad 443 \quad 240.62
$$

means that 25 is the primary pretender for the bases that are 4 th roots of 0 or $1(\bmod 25)$ that have not already been coped with, that the first such base is 443 , and that 1 in every 240.62 bases has 25 for its primary pretender (in fact, 16 in every 3465 bases).

Another example is "else 169 if $b^{12} \equiv 0$ or $1(\bmod 169)$ ", i.e., if $b \equiv \pm 19^{e}$ $(\bmod 169)$, for $1 \leq e \leq 6$ where the cases $e=6(b \equiv \pm 1), e=3(b \equiv \pm 70)$, and $e=2$ or $4(b \equiv \pm 23$ or \pm 22$)$ have already been preempted by $q_{b}=26$ or 39 , by 65 , and by 91 respectively. 
Table 2. $q_{b} \geq 22$ for 32 residue classes $\bmod 1260,2 \leq b \leq 51602$

\begin{tabular}{|c|c|c|c|c|c|c|c|c|c|c|c|c|c|c|c|c|}
\hline$b=$ & 2 & 23 & 38 & 47 & 122 & 158 & 227 & 263 & 338 & 347 & 362 & 383 & 443 & 527 & 542 & 563 \\
\hline 0 & 341 & 22 & 38 & 46 & 22 & 158 & 49 & 33 & 26 & 87 & 33 & 382 & 25 & 33 & 91 & 91 \\
\hline 1 & 26 & 91 & 22 & 25 & 25 & 25 & 65 & 185 & 34 & 22 & 91 & 25 & 26 & 38 & 34 & 57 \\
\hline 2 & 26 & 25 & 91 & 34 & 38 & 26 & 82 & 22 & 113 & 94 & 22 & 33 & 39 & 22 & 145 & 46 \\
\hline 3 & 25 & 49 & 22 & 33 & 49 & 22 & 25 & 25 & 25 & 85 & 38 & 46 & 33 & 25 & 33 & 25 \\
\hline 4 & 121 & 122 & 49 & 85 & 26 & 46 & 46 & 22 & 33 & 65 & 22 & 22 & 91 & 22 & 25 & 26 \\
\hline 5 & & 51 & 133 & 22 & 26 & 22 & 26 & 34 & 65 & 34 & 133 & 26 & 22 & 145 & 22 & 33 \\
\hline 6 & 38 & 34 & 51 & 25 & 25 & 25 & 26 & 91 & 22 & 25 & 34 & 22 & 65 & 26 & 91 & 62 \\
\hline 7 & 22 & 25 & 34 & 22 & 33 & 65 & 39 & 38 & 38 & 85 & 25 & 39 & 22 & 26 & 22 & 22 \\
\hline 8 & 25 & 561 & 25 & 26 & 57 & 58 & 22 & 25 & 22 & 26 & 46 & 327 & 34 & 25 & 26 & 25 \\
\hline 9 & 22 & 22 & 62 & 39 & 22 & 121 & 86 & 82 & 51 & 26 & 141 & 38 & 65 & 34 & 25 & 22 \\
\hline 10 & 6 & 26 & 33 & 51 & 49 & 49 & 22 & 38 & 34 & 22 & 26 & 91 & 25 & 91 & 34 & 49 \\
\hline 11 & 58 & 22 & 26 & 25 & 22 & 25 & 65 & 33 & 62 & 25 & 26 & 25 & 91 & 33 & 38 & 205 \\
\hline 12 & 49 & 25 & 22 & 58 & 145 & 33 & 85 & 91 & 26 & 22 & 25 & 46 & 49 & 65 & 49 & 65 \\
\hline 13 & 25 & 91 & 25 & 65 & 58 & 46 & 25 & 22 & 25 & 49 & 22 & 33 & 26 & 22 & 91 & 25 \\
\hline 14 & 26 & 34 & 22 & 33 & 91 & 22 & 34 & 49 & 39 & 34 & 49 & 65 & 26 & 62 & 25 & 38 \\
\hline 15 & 26 & 57 & 34 & 169 & 46 & 26 & 62 & 22 & 33 & 38 & 22 & 22 & 25 & 22 & 145 & 74 \\
\hline 16 & 33 & 85 & 38 & 22 & 25 & 22 & 38 & 26 & 74 & 25 & 62 & 25 & 22 & 91 & 22 & 26 \\
\hline 17 & 62 & 25 & 65 & 85 & 26 & 49 & 33 & 39 & 22 & 65 & 25 & 22 & 34 & 34 & 65 & 26 \\
\hline 18 & 22 & 33 & 25 & 22 & 26 & 38 & 25 & 25 & 25 & 57 & 85 & 26 & 22 & 25 & 22 & 22 \\
\hline 19 & 49 & 62 & 38 & 34 & 39 & 87 & 22 & 91 & 22 & 33 & 38 & 26 & 49 & 26 & 25 & 133 \\
\hline 20 & 22 & 22 & 6 & 26 & 22 & 62 & 39 & 58 & 85 & 49 & 91 & 39 & 25 & 26 & 85 & 22 \\
\hline 21 & 94 & 142 & 33 & 25 & 25 & 25 & 22 & 49 & 65 & 22 & 49 & 25 & 57 & 39 & 26 & 91 \\
\hline 22 & 38 & 22 & 57 & 39 & 22 & 106 & 51 & 33 & 49 & 26 & 25 & 91 & 65 & 33 & 26 & 65 \\
\hline 23 & 25 & 26 & 22 & 46 & 65 & 33 & 25 & 25 & 25 & 22 & 26 & 85 & 85 & 25 & 39 & 25 \\
\hline 24 & 51 & 39 & 26 & 38 & 34 & 49 & 65 & 22 & 133 & 82 & 22 & 33 & 46 & 22 & 25 & 49 \\
\hline 25 & 34 & 58 & 22 & 33 & 145 & 22 & & 46 & 26 & 91 & 39 & 38 & 25 & 51 & 33 & 34 \\
\hline 26 & 49 & 74 & 39 & 25 & 25 & 25 & 301 & 22 & 26 & 25 & 22 & 22 & 26 & 22 & 49 & 91 \\
\hline 27 & 26 & 25 & 145 & 22 & 57 & 22 & 46 & 121 & 34 & 49 & 25 & 65 & 22 & 46 & 22 & 33 \\
\hline 28 & 25 & 38 & 25 & 34 & 62 & 26 & 25 & 25 & 22 & 46 & 49 & 22 & 39 & 25 & 133 & 25 \\
\hline 29 & 22 & 33 & 91 & 22 & 33 & 39 & 62 & 26 & 49 & 87 & 65 & 51 & 22 & 86 & 22 & 22 \\
\hline 30 & 82 & 218 & 65 & 49 & 26 & 529 & 22 & 34 & 22 & 33 & 62 & 34 & 25 & 91 & 38 & 26 \\
\hline 31 & 22 & 22 & 226 & 25 & 22 & 25 & 26 & 87 & 65 & 25 & 46 & 25 & 91 & 62 & 91 & 22 \\
\hline 32 & 91 & 25 & 33 & 74 & 39 & 34 & 22 & 57 & 58 & 22 & 25 & 26 & 65 & 26 & 58 & 217 \\
\hline 33 & 25 & 22 & 25 & 26 & 22 & 65 & 25 & 25 & 25 & 185 & 33 & 39 & 49 & 25 & 49 & 25 \\
\hline 34 & 62 & 57 & 22 & 26 & 91 & 33 & 111 & 46 & 65 & 22 & 91 & 205 & 34 & 34 & 25 & 91 \\
\hline 35 & 85 & 26 & 38 & 39 & 91 & 133 & 38 & 22 & 34 & 26 & 22 & 33 & 25 & 22 & 26 & 65 \\
\hline 36 & 65 & 26 & 22 & 25 & 25 & 22 & 46 & 86 & 49 & 25 & 26 & 25 & 33 & 85 & 33 & 38 \\
\hline 37 & 46 & 25 & 26 & 46 & 86 & 38 & 65 & 22 & 33 & 46 & 22 & 22 & 38 & 22 & 85 & 58 \\
\hline 38 & 25 & 49 & 25 & 22 & 46 & 22 & 25 & 25 & 25 & 58 & 38 & 34 & 22 & 25 & 22 & 25 \\
\hline 39 & 133 & 51 & 39 & 65 & 65 & 133 & 33 & 34 & 22 & 34 & 87 & 22 & 26 & 38 & 25 & 46 \\
\hline 40 & 22 & 33 & 51 & 22 & 33 & 26 & 34 & 58 & 39 & 62 & 34 & 49 & 22 & 133 & 22 & 22 \\
\hline
\end{tabular}


Table 2 (cont.)

\begin{tabular}{|c|c|c|c|c|c|c|c|c|c|c|c|c|c|c|c|c|}
\hline$b=$ & 578 & 662 & 698 & 758 & 767 & 803 & 842 & 878 & 887 & 947 & 983 & 1067 & 1082 & 1103 & 1118 & 1202 \\
\hline 0 & 34 & 39 & 34 & 33 & 26 & 22 & 58 & 259 & 91 & 22 & 65 & 22 & 25 & 38 & 25 & 169 \\
\hline 1 & 22 & 34 & 22 & 25 & 39 & 91 & 22 & 49 & 38 & 25 & 25 & 26 & 33 & 34 & 39 & 46 \\
\hline 2 & 38 & 25 & 25 & 22 & 38 & 22 & 49 & 33 & 25 & 51 & 34 & 22 & 26 & 91 & 34 & 51 \\
\hline 3 & 91 & 91 & 22 & 26 & 91 & 58 & 69 & 34 & 26 & 34 & 22 & 74 & 22 & 33 & 62 & 25 \\
\hline 4 & 25 & 49 & 38 & 22 & 25 & 25 & 25 & 22 & 39 & 82 & 38 & 25 & 39 & 25 & 85 & 49 \\
\hline 5 & 26 & 33 & 91 & 39 & 57 & 65 & 65 & 74 & 85 & 133 & 22 & 58 & 22 & 22 & 25 & 22 \\
\hline 6 & 26 & 365 & 46 & 25 & 22 & 51 & 62 & 22 & 33 & 25 & 25 & 38 & 49 & 57 & 33 & 26 \\
\hline 7 & 39 & 22 & 25 & 91 & 51 & 34 & 91 & 26 & 25 & 33 & 26 & 133 & 91 & 22 & 38 & 22 \\
\hline 8 & 33 & 46 & 26 & 91 & 22 & 341 & 33 & 39 & 22 & 74 & 26 & 142 & 65 & 91 & 22 & 25 \\
\hline 9 & 25 & 22 & 26 & 46 & 25 & 25 & 25 & 25 & 85 & 22 & 39 & 25 & 123 & 25 & 91 & 85 \\
\hline 10 & 22 & 38 & 33 & 38 & 82 & 26 & 22 & 46 & 22 & 26 & 34 & 51 & 25 & 26 & 22 & 74 \\
\hline 11 & 65 & 26 & 58 & 25 & 69 & 22 & 39 & 51 & 65 & 22 & 25 & 22 & 51 & 26 & 34 & 34 \\
\hline 12 & 22 & 25 & 22 & 34 & 26 & 38 & 22 & 34 & 25 & 39 & 33 & 57 & 33 & 39 & 26 & 38 \\
\hline 13 & 62 & 39 & 91 & 22 & 26 & 22 & 34 & 33 & 49 & 49 & 65 & 22 & 38 & 93 & 26 & 25 \\
\hline 14 & 25 & 451 & 22 & 85 & 25 & 25 & 25 & 25 & 118 & 91 & 22 & 25 & 22 & 25 & 39 & 33 \\
\hline 15 & 85 & 62 & 82 & 22 & 33 & 34 & 38 & 22 & 26 & 91 & 118 & 39 & 25 & 91 & 25 & 38 \\
\hline 16 & 91 & 33 & 51 & 25 & 34 & 94 & 49 & 91 & 26 & 25 & 22 & 87 & 22 & 22 & 91 & 22 \\
\hline 17 & 34 & 25 & 25 & 26 & 22 & 82 & 123 & 22 & 25 & 106 & 46 & 85 & 39 & 133 & 33 & 133 \\
\hline 18 & 26 & 22 & 91 & 39 & 38 & 46 & 38 & 85 & 133 & 33 & 51 & 49 & 85 & 22 & 5 & 22 \\
\hline 19 & 25 & 146 & 91 & 106 & 22 & 25 & 25 & 25 & 22 & 46 & 34 & 25 & 57 & 25 & 22 & 26 \\
\hline 20 & 39 & 22 & 38 & 51 & 46 & 33 & 58 & 26 & 38 & 22 & 26 & 33 & 25 & 58 & 25 & 34 \\
\hline 21 & 22 & 74 & 26 & 25 & 38 & 49 & 22 & 39 & 22 & 25 & 25 & 85 & 65 & 86 & 22 & 278 \\
\hline 22 & 91 & 25 & 25 & 33 & 49 & 22 & 26 & 49 & 25 & 22 & 39 & 22 & 85 & 38 & 91 & 91 \\
\hline 23 & 22 & 121 & 22 & 69 & 91 & 26 & 22 & 91 & 57 & 26 & 33 & 91 & 3 & 26 & 91 & 25 \\
\hline 24 & 25 & 26 & 49 & 22 & 25 & 22 & 25 & 25 & 34 & 26 & 91 & 22 & 65 & 25 & 133 & 46 \\
\hline 25 & 51 & 26 & 22 & 65 & 26 & 62 & 206 & 65 & 91 & 39 & 22 & 38 & 22 & 33 & 25 & 33 \\
\hline 26 & 34 & 34 & 57 & 22 & 26 & 91 & 69 & 22 & 85 & 25 & 25 & 26 & 86 & 51 & 26 & 85 \\
\hline 27 & 57 & 25 & 25 & 85 & 39 & 46 & 91 & 341 & 25 & 49 & 22 & 26 & 22 & 22 & 39 & 22 \\
\hline 28 & 46 & 85 & 133 & 74 & 22 & 49 & 65 & 22 & 26 & 34 & 133 & 34 & 26 & 91 & 33 & 25 \\
\hline 29 & 25 & 22 & 46 & 26 & 25 & 25 & 25 & 25 & 26 & 33 & 65 & 25 & 26 & 22 & 38 & 22 \\
\hline 30 & 33 & 65 & 65 & 26 & 22 & 85 & 33 & 91 & 22 & 85 & 91 & 91 & 25 & 106 & 22 & 85 \\
\hline 31 & 26 & 22 & 49 & 25 & 65 & 33 & 65 & 38 & 86 & 22 & 25 & 33 & 169 & 86 & 65 & 26 \\
\hline 32 & 22 & 25 & 25 & 38 & 321 & 34 & 22 & 26 & 22 & 57 & 206 & 49 & 38 & 46 & 22 & 26 \\
\hline 33 & 39 & 65 & 51 & 33 & 34 & 22 & 91 & 26 & 34 & 22 & 26 & 22 & 74 & 85 & 49 & 25 \\
\hline 34 & 22 & 51 & 22 & 91 & 25 & 25 & 22 & 25 & 49 & 49 & 26 & 25 & 33 & 25 & 62 & 38 \\
\hline 35 & 86 & 34 & 26 & 22 & 85 & 22 & 26 & 33 & 69 & 202 & 39 & 22 & 25 & 34 & 25 & 91 \\
\hline 36 & 85 & 69 & 22 & 25 & 49 & 26 & 26 & 49 & 38 & 25 & 22 & 34 & 22 & 26 & 34 & 33 \\
\hline 37 & 65 & 25 & 25 & 22 & 33 & 39 & 38 & 22 & 25 & 26 & 91 & 86 & 34 & 26 & 169 & 34 \\
\hline 38 & 87 & 26 & 49 & 34 & 26 & 178 & 34 & 65 & 74 & 39 & 22 & 85 & 22 & 22 & 26 & 22 \\
\hline 39 & 25 & 39 & 38 & 82 & 22 & 25 & 25 & 22 & 33 & 158 & 38 & 25 & 85 & 25 & 25 & 49 \\
\hline 40 & 38 & 22 & 58 & 91 & 38 & 51 & 57 & 94 & 169 & 33 & 46 & 26 & 25 & 22 & 25 & 22 \\
\hline
\end{tabular}


Table 3. The first base and rarity of the 132 primary pretenders

\begin{tabular}{|c|c|c|c|c|c|c|c|}
\hline$q$ & $\begin{array}{l}\text { roots } \\
k \operatorname{th}(m)\end{array}$ & $\begin{array}{l}\text { first } \\
\text { base }\end{array}$ & $\begin{array}{l}\text { rarity } \\
\text { one in }\end{array}$ & $q$ & $\begin{array}{l}\text { roots } \\
k \operatorname{th}(m)\end{array}$ & $\begin{array}{l}\text { first } \\
\text { base }\end{array}$ & $\begin{array}{l}\text { rarity } \\
\text { one in }\end{array}$ \\
\hline 4 & $1 \mathrm{st}(4)$ & 0 & 2 & 159 & $2 \mathrm{nd}(53)$ & 94763 & 83341.92 \\
\hline 6 & $1 \mathrm{st}(3)$ & 3 & 3 & 166 & $1 \mathrm{st}(83)$ & 247838 & 69173.80 \\
\hline 9 & 2nd(9) & 26 & 18 & 169 & $12 \operatorname{th}(169)$ & 1202 & 22203.93 \\
\hline 10 & $1 \mathrm{st}(5)$ & 11 & 22.5 & 177 & $2 \mathrm{nd}(59)$ & 111863 & 105468.69 \\
\hline 14 & $1 \mathrm{st}(7)$ & 14 & 52.5 & 178 & 1 st(89) & 48683 & 83809.94 \\
\hline 15 & $2 \operatorname{nd}(5)$ & 59 & 63 & 183 & $2 \mathrm{nd}($ & 186842 & 113673.26 \\
\hline 21 & $2 \mathrm{nd}(7)$ & 83 & 157.5 & 185 & $4 \operatorname{th}(37)$ & 1523 & 33318.02 \\
\hline 22 & $1 \mathrm{st}(11)$ & 23 & 216.56 & 194 & 1st(97) & 58298 & 100995.26 \\
\hline 25 & $4 \operatorname{th}(25)$ & 443 & 240.62 & 201 & $2 \mathrm{nd}(67)$ & 86027 & 138204.04 \\
\hline 26 & 1st(13) & 338 & 391.01 & 202 & 1st(101) & 45047 & 109051.62 \\
\hline 33 & $2 \mathrm{nd}(11)$ & 263 & 639.84 & 205 & $4 \operatorname{th}($ & 14423 & 41858.20 \\
\hline 34 & 1st(17) & 578 & 679.83 & 206 & $1 \mathrm{st}(1$ & 32342 & 119760.96 \\
\hline 38 & $1 \mathrm{st}(19)$ & 38 & 861.12 & 213 & & 53462 & 163633.79 \\
\hline 39 & $2 \mathrm{nd}(13)$ & 662 & 1114.39 & 214 & $1 \mathrm{st}(1$ & 79502 & 128741.29 \\
\hline 46 & $1 \mathrm{st}(23)$ & 47 & 1281.55 & 217 & & 40883 & 17165.50 \\
\hline 49 & $6 \operatorname{th}(49)$ & 227 & 854.36 & 218 & $1 \mathrm{st}($ & 37823 & 155920.00 \\
\hline 51 & $2 \mathrm{nd}(17)$ & 3467 & 2135.92 & 219 & $2 \mathrm{nd}($ & 169067 & 206921.87 \\
\hline 57 & $2 \mathrm{nd}(19)$ & 1823 & 2593.62 & 226 & $1 \mathrm{st}(1$ & 39098 & 167015.51 \\
\hline 58 & $1 \mathrm{st}(29)$ & 842 & 2350.46 & 237 & & 141962 & 231715.22 \\
\hline 62 & 1 st (31) & 4898 & 2698.68 & 249 & $2 \mathrm{nd}($ & 357563 & 246959.64 \\
\hline 65 & $4 \operatorname{th}(1$ & & 930.58 & 254 & 1st(127) & 232538 & 196024.21 \\
\hline 69 & $2 \mathrm{nd}(23)$ & 4622 & 4885.55 & 259 & $6 \operatorname{th}($ & 878 & 25091.09 \\
\hline 74 & 1st(37) & 4847 & 4519.13 & 262 & $1 \mathrm{st}(131)$ & 162047 & 234781.00 \\
\hline 82 & $1 \mathrm{st}(41)$ & 2747 & 5293.84 & 265 & $4 \operatorname{th}($ & 98663 & 91000.38 \\
\hline 85 & $4 \operatorname{th}(17)$ & 4127 & 1900.35 & 267 & 2nd(89) & 232823 & 329876.41 \\
\hline 86 & 1 st(43) & 11567 & 6809.60 & 274 & 1st(137) & 112478 & 262750.39 \\
\hline 87 & 2nd(29) & 347 & 8968.74 & 278 & 1st(139) & 27662 & 270535.59 \\
\hline 91 & $6 \operatorname{th}(13)$ & 542 & 689.90 & 289 & $16 \operatorname{th}(289)$ & 197138 & 67140.22 \\
\hline 93 & $2 \mathrm{nd}(31)$ & 17483 & 20007.20 & 291 & $2 \mathrm{nd}(97)$ & 124547 & 398645.06 \\
\hline 94 & $1 \mathrm{st}(47)$ & 2867 & 16791.76 & 298 & 1st(149) & 142742 & 315947.41 \\
\hline 106 & $1 \mathrm{st}(53)$ & 22367 & 19776.96 & 301 & $6 \operatorname{th}(43)$ & 32987 & 42986.04 \\
\hline 111 & 2nd(37) & 43067 & 27144.85 & 302 & 1st(151) & 150698 & 360605.13 \\
\hline 118 & 1 st(59) & 18527 & 23552.15 & 303 & $2 \mathrm{nd}(101)$ & 485102 & 479193.40 \\
\hline 121 & $10 \operatorname{th}(121)$ & 5042 & 9090.30 & 305 & $4 \operatorname{th}(61)$ & 287138 & 141802.12 \\
\hline 122 & $1 \mathrm{st}(61)$ & 5063 & 27725.42 & 309 & 2nd(103) & 79103 & 511500.54 \\
\hline 123 & 2nd(41) & 12422 & 36653.95 & 314 & 1st(157) & 115238 & 401527.92 \\
\hline 129 & $2 \mathrm{nd}(43)$ & 66047 & 39547.68 & 321 & $2 \mathrm{nd}(107)$ & 41087 & 544005.57 \\
\hline 133 & $6 \operatorname{th}(19)$ & 2858 & 3954.76 & 326 & 1 st(163) & 296987 & 426312.06 \\
\hline 134 & 1st(67) & 87302 & 44161.58 & 327 & 2nd(109) & 10463 & 566650.81 \\
\hline 141 & $2 \mathrm{nd}(47)$ & 11702 & 61146.80 & 334 & 1st(167) & 127922 & 446371.15 \\
\hline 142 & $1 \mathrm{st}(71)$ & 11147 & 49334.35 & 339 & 2nd(113) & 851567 & 600572.10 \\
\hline 145 & $4 \operatorname{th}(29)$ & 3062 & 18589.75 & 341 & $10 \operatorname{th}(31)$ & 2 & 16379.23 \\
\hline 146 & $1 \mathrm{st}(73)$ & 24602 & 56543.84 & 346 & 1st(173) & 846662 & 708402.09 \\
\hline 158 & 1st(79) & 158 & 62914.98 & 358 & 1st(179) & 257402 & 741543.71 \\
\hline
\end{tabular}


Table 3 (cont.)

\begin{tabular}{|c|c|c|c|c|c|c|c|}
\hline$q$ & $\begin{array}{l}\text { roots } \\
k \operatorname{th}(m)\end{array}$ & $\begin{array}{l}\text { first } \\
\text { base }\end{array}$ & $\begin{array}{l}\text { rarity } \\
\text { one in }\end{array}$ & $q$ & $\begin{array}{l}\text { roots } \\
k \operatorname{th}(m)\end{array}$ & $\begin{array}{l}\text { first } \\
\text { base }\end{array}$ & $\begin{array}{l}\text { rarity } \\
\text { one in }\end{array}$ \\
\hline 361 & $18 \operatorname{th}(361)$ & 58727 & 159201.47 & 471 & $2 \mathrm{nd}(157)$ & 2264567 & 2457680.13 \\
\hline 362 & $1 \mathrm{st}(181)$ & 1050887 & 800429.64 & 478 & 1st(239) & 6085658 & 1907095.94 \\
\hline 365 & $4 \operatorname{th}(73)$ & 8222 & 313017.17 & 481 & $12 \operatorname{th}(37)$ & 108803 & 112655.45 \\
\hline 381 & 2nd(127) & 923162 & 1150798.45 & 482 & $1 \mathrm{st}(241)$ & 2252387 & 2262497.08 \\
\hline 382 & 1st(191) & 383 & 886300.41 & 485 & $4 \operatorname{th}(97)$ & 968567 & 889852.41 \\
\hline 386 & 1st(193) & 470342 & 905058.10 & 489 & $2 \mathrm{nd}(163)$ & 3166763 & 3114483.44 \\
\hline 393 & $2 \mathrm{nd}(131)$ & 480638 & 1222539.21 & 501 & $2 \mathrm{nd}(167)$ & 4881242 & 3211811.04 \\
\hline 394 & 1st(197) & 384347 & 940782.12 & 502 & 1st(251) & 1738427 & 2457818.82 \\
\hline 398 & 1st(199) & 278402 & 960080.22 & 505 & $4 \operatorname{th}(101)$ & 2128262 & 967334.31 \\
\hline 411 & $2 \mathrm{nd}(137)$ & 786242 & 1315845.99 & 511 & $6 \operatorname{th}(73)$ & 210962 & 342597.56 \\
\hline 417 & 2nd(139) & 303158 & 1345305.23 & 514 & 1st(257) & 2338187 & 2751486.73 \\
\hline 422 & 1st(211) & 231467 & 1043600.74 & 519 & 2nd(173) & 1150103 & 3690229.26 \\
\hline 427 & $6 \operatorname{th}(61)$ & 149558 & 139812.54 & 526 & 1 st $(263)$ & 256163 & 2854500.87 \\
\hline 445 & $4 \operatorname{th}(89)$ & 739022 & 462456.86 & 529 & $22 \mathrm{nd}(529)$ & 37958 & 503092.10 \\
\hline 446 & 1 st $(223)$ & 592958 & 1227712.86 & 537 & 2nd(179) & 7345622 & 4047604.68 \\
\hline 447 & 2nd(149) & 141698 & 1633246.98 & 538 & 1st $(269)$ & 2735462 & 3093197.89 \\
\hline 451 & $10 \operatorname{th}(41)$ & 18302 & 50339.80 & 542 & $1 \mathrm{st}(271)$ & 183467 & 3139537.94 \\
\hline 453 & $2 \mathrm{nd}(151)$ & 10009487 & 2143037.38 & 543 & $2 \mathrm{nd}(181)$ & 4503098 & 4178269.82 \\
\hline 454 & 1st(227) & 283523 & 1643477.99 & 545 & $4 \operatorname{th}(109)$ & 4453598 & 1244091.57 \\
\hline 458 & 1st (229) & 277778 & 1672695.37 & 553 & $6 \operatorname{th}(79)$ & 281738 & 454571.92 \\
\hline 466 & $1 \mathrm{st}(233)$ & 860702 & 1716907.59 & 554 & 1st(277) & 581423 & 3497678.40 \\
\hline 469 & $6 \operatorname{th}(67)$ & 473987 & 237840.01 & 561 & $1 \mathrm{st}(1)$ & 10103 & 25437.66 \\
\hline
\end{tabular}

The largest first base is 10009487 , for $q=453$, while the greatest rarity is that of $q=519$.

The paper was prompted by the table of pseudoprimes to various bases given by Albert H. Beiler on p. 42 of his Recreations in the Theory of Numbers, Dover, New York, 1964.

Department of Mathematics Princeton University

Princeton, New Jersey 08544

U.S.A.

E-mail: conway@math.princeton.edu william@math.princeton.edu
AT\&T Bell Laboratories Mathematical Sciences Research Center Murray Hill, New Jersey 07974 E-mail: njas@research.att.com

Department of Mathematics

The University of Calgary

Calgary, Alberta

Canada T2N 1N4

E-mail: rkg@cpsc.ucalgary.ca 